Georgian Mathematical Journal

1(1994), No. 5, 537-559

\title{
FRACTIONAL TYPE OPERATORS IN WEIGHTED GENERALIZED HÖLDER SPACES
}

\author{
S.G. SAMKO AND Z.U. MUSSALAEVA
}

\begin{abstract}
Weighted Zygmund type estimates are obtained for the continuity modulus of some convolution type integrals. In the case of fractional integrals this is strengthened to a result on isomorphism between certain weighted generalized Hölder type spaces.
\end{abstract}

\section{INTRODUCTION}

A great number of results is known concerning boundedness of convolution type operators in spaces of summable functions, including the weighted case. In the spaces of continuous functions such as $H_{0}^{\omega}(\rho)$ the convolution type operators are less investigated. The goal of this paper is to fill a gap to a certain extent in investigations of such a kind.

We consider here the Volterra convolution type operators

$$
K \varphi=\int_{a}^{x} k(x-t) \varphi(t) d t, \quad a<x<b,
$$

in the weighted generalized Hölder spaces $H_{0}^{\omega}(\rho)$ (see definitions in Sec.2), $-\infty<a<b<\infty$. The kernel $k(x)$ is assumed to be close in a sense to a power function.

The result of the type

$$
K: H_{0}^{\omega}(\rho) \rightarrow H_{0}^{\omega_{1}}(\rho)
$$

for certain characteristic functions $\omega(h)$ and $\omega_{1}(h)$ was earlier known in the case of the power kernel $k(x)=x^{\alpha-1}, 0<\alpha<1$, and a power weight function $\rho(t)$. We deal here with arbitrary kernels and weights, i.e. not necessarily power ones.

We introduce a certain class $V_{\lambda}$ of kernels and the class $w_{\mu}$ of weight functions for which we manage to give the weighted Zygmund type estimate, that is, to estimate the modulus of continuity $\omega\left(\rho K_{\varphi}, h\right)$ by the modulus of 
continuity $\omega(\rho \varphi, h)$. This estimate provides the general result of the type (1.2).

In the case of purely power kernel, i.e. in the case of the fractional integration operator

$$
I_{a+\varphi}^{\alpha} \varphi=\frac{1}{\Gamma(\alpha)} \int_{a}^{x}(x-t)^{\alpha-1} \varphi(t) d t, \quad 0<\alpha<1,
$$

the result (1.2) is extended to isomorphism:

$$
I_{a^{+}}^{\alpha}\left[H_{0}^{\omega}(\rho)\right]=H_{0}^{\omega_{\alpha}}(\rho)
$$

with $\omega_{\alpha}(h)=h^{\alpha} \omega(h)$. This is achieved by the preliminary derivation of Zygmund type estimate for fractional differentiation. The latter is treated in a difference form due to A.Marchaud [9] and G.H.Hardy and J.E.Littlewood [2]: (See [17], Sec.13, in this connection.)

$$
\begin{gathered}
D_{a+}^{\alpha} f(x)=\frac{f(x)}{\Gamma(1-\alpha)(x-a)^{\alpha}}+\frac{\alpha}{\Gamma(1-\alpha)} \int_{a}^{x} \frac{f(x)-f(t)}{(x-t)^{1+\alpha}} d t, \\
0<\alpha<1 .
\end{gathered}
$$

The paper is organized as follows. In Sec.2 we give necessary preliminaries. Sec.3 contains Zygmund type estimates for the operator (1.2) in the case of kernels in $V_{\lambda}$ in the non-weighted case first (Theorem 1) and afterwards in the weighted case (Theorem 2). In Theorem 3 we give conditions of Zygmund-Bari-Stechkin type on a characteristic function $\omega(h)$ guaranteeing the result (1.2) for $k(x) \in V_{\lambda}$ and weighted functions in $w_{\mu}$. The characteristic function $\omega_{1}(h)$ in (1.2) proves to be equal to $h k(h) \omega(h)$. We note corollary of Theorem 3 for $k(x)=x^{\alpha-1}\left(\ln \frac{\gamma}{x}\right)^{\beta}, \gamma>b-a$.

In Sec.4 we establish the weighted Zygmund type estimate for $D_{a+}^{\alpha} f$ with a weight function in $w_{\mu}$ (Theorem 4). We prove the assertion $D_{a+}^{\alpha}$ : $H_{0}^{\omega}(\rho) \rightarrow H_{0}^{\omega_{-\alpha}}(\rho)$ with $\omega_{-\alpha}(h)=h^{-\alpha} \omega(h)$ under appropriate assumptions on $\omega(h)$ and $\rho(x)$ (Theorem 5).

As a corollary of Theorems 3 and 5 we give conditions for validity of the second index law of E.R.Love within the framework of the spaces $H_{0}^{\lambda}\left(x^{\mu}\right)$

Finally, in Sec.5 we prove the isomorphism (1.4) (Theorem 6).

Presented theorems generalize the results of the papers [10]-[12],[18], where the power case for both $k(x)=x^{\alpha-1}$ and $\rho(x)=(x-a)^{\alpha}$ was considered. The presentation of the results of [10] in the non-weighted case can be also found in [18], Sec.13. Note that in [12] the case $\rho(x)=(x-$ $a)^{\mu}(b-x)^{v}$ was also considered, not contained in the results of the present paper. The origin of the statement (1.4) is the classical result by G.H.Hardy and J.E.Littlewood [2] for the fractional integration concerning the case 
$\omega(h)=h^{\lambda}, \rho(x) \equiv 1, \alpha+\lambda<1$. (As for the case $\omega(h)=\prod_{k=1}^{n}\left|x-x_{k}\right|^{\mu_{k}}$, see [13] and [17]. Sec.13.)

We also note the papers [4], [5] where Zygmund type estimates are given for the fractional integrodifferentiation in the case of $L_{p}$-moduli of continuity.

The question we finally note as open is whether $I_{a+}^{\alpha}\left[H_{0}^{\omega}(\rho)\right]=H_{0}^{\omega}(\rho)$ in the case of purely imaginary $\alpha$, under the appropriate assumptions on $\omega(h)$ and $\rho(x)$. We refer to the paper [7] by E.R.Love concerning such fractional integrals (see also [17], Sec.2, $n^{0} 4$ ).

\section{Preliminaries}

We follow the papers [14], [15] in the definitions below.

Definition 1. We say that $\psi(x) \in W_{\mu}=W_{\mu}([0, l])$ if $\psi(x) \in C([0, l])$, $\psi(0)=0, \psi(x)>0$ for $x>0, \psi(x)$ is almost increasing, while $\psi(x) / x^{\mu}$ is almost decreasing and there exists a constant $c>0$ such that

$$
\left|\frac{\psi(x)-\psi(y)}{x-y}\right| \leq c \frac{\psi\left(x^{*}\right)}{x^{*}}, \quad x^{*}=\max (x, y) .
$$

We remind that a non-negative function $\psi(x), 0 \leq x \leq l, 0<l \leq \infty$, is called almost increasing (decreasing) if $\psi(x) \leq c \psi(y)$ for all $x \leq y(x \geq y$, resp.), this notion being due to S.Bernstein.

Definition 2. We say that $\psi(x) \in W_{\mu}^{*}$ if $\psi(x) \in W_{\mu}$ and $\psi(x) / x^{\mu-\varepsilon}$ is almost increasing for all $\varepsilon>0$.

We shall also need the following modification of the Definition 2.

Definition 3. We say that a non-negative function $k(x)$ on $[0, l]$ belongs to the class $V_{\lambda}, \lambda>0$ if

i) $k(x) \not \equiv 0, x^{\lambda} k(x)$ is almost increasing and $\left.x^{\lambda} k(x)\right|_{x=0}=0$;

ii) there exists $\varepsilon, 0<\varepsilon<\lambda$, such that $x^{\lambda-\varepsilon} k(x)$ is almost decreasing;

iii) there exists $c>0$ such that

$$
\left|\frac{k(x)-k(y)}{x-y}\right| \leq c \frac{k\left(x^{*}\right)}{x^{*}}, \quad x^{*}=\max (x, y) .
$$

Remark 1. $x^{\lambda} k(x) \in W_{\lambda}^{*} \Rightarrow k(x) \in V_{\lambda}$ and $k(x) \in V_{\lambda} \Rightarrow k(x) \in W_{\lambda}$.

Remark 2. If the almost monotonicity in Definitions 1 and 3 is replaced by the usual monotonicity, then conditions (2.1) and (2.2) are satisfied automatically.

Indeed, let us prove e.g. (2.1), following [14]. If $\varphi(x) / x^{\mu}$ is decreasing, so that $1-\varphi(x) / \varphi(y) \leq 1-x^{\mu} / y^{\mu}$ for $y \geq x$, then $\varphi(y)-\varphi(x) \leq \frac{y^{\mu}-x^{\mu}}{y^{\mu}} \varphi(y)$. Since $y^{\mu}-x^{\mu} \leq c(y-x) y^{\mu-1}$, we obtain (2.1).

Definition 4 ([1]). A non-negative function $\varphi(t)$ on $[0, l]$ belongs to Zygmund class $Z=Z([0, l])$ if $\int_{0}^{h} \frac{\varphi(t)}{t} d t \leq c \varphi(h), 0 \leq h \leq l$. 
Definition 5 ([1]). A non-negative function $\varphi(t)$ belongs to Zygmund class $Z_{1}=Z_{1}([0, l])$ if $\int_{h}^{l} \frac{\varphi(t)}{t^{2}} d t \leq c \frac{\varphi(h)}{h}$.

Definition 6. A function $\varphi(x)$ belongs to the generalized Hölder space $H^{\omega}=H^{\omega}([a, b])$ if

$$
\omega(\varphi, h) \stackrel{\text { def }}{=} \sup _{0<t<h} \sup _{x, x+t \in[a, b]}|\varphi(x+t)-\varphi(x)| \leq c \omega(h),
$$

where $\omega(h)$ is a given positive function on $[0, l], \omega(0)=0$; we set $\|\varphi\|_{H^{\omega}}=$ $\|\varphi\|_{C}+\sup _{h>0}[\omega(\varphi, h) / \omega(h)]$.

By $H_{0}^{\omega}$ we denote the subspace of functions in $H^{\omega}$ which vanish at $x=a$.

The function $\omega(h)$ is called a characteristic of the space $H^{\omega}$.

Definition 7. By $H_{0}^{\omega}(\rho)$ we denote the space of functions $f(x)$ such that $\rho(x) f(x) \in H_{0}^{\omega},\|f\|_{H_{0}^{\omega}(\rho)}=\|\rho f\|_{H_{0}^{\omega}}$, where $\rho(x)$ is a non-negative weight function.

In the sequel we shall use the following inequalities:

$1)$ if $\omega(\varphi, h)$ is the continuity modulus $(2.3)$, then

$$
\frac{\omega(\varphi, x)}{x} \leq c \frac{\omega(\varphi, y)}{y}, \quad x \geq y ;
$$

2) if $0<\alpha<1$, then

$$
\frac{\omega(\varphi, h)}{h^{\alpha}} \leq c \int_{0}^{h} \frac{\omega(\varphi, t)}{t^{1+\alpha}} d t
$$

3) if $\psi(x) \in W_{\mu}$, then

$$
\psi(x) \leq c\left(\frac{x}{y}\right)^{\mu} \psi(y), \quad x \geq y ;
$$

4) if $\psi(x) \in W_{\mu}$ with $0<\mu<1$, then (2.1) holds with $x^{*}$ replaced both by $x$ and $y$ :

$$
\begin{aligned}
& \left|\frac{\psi(x)-\psi(y)}{x-y}\right| \leq c \frac{\psi(x)}{x} \\
& \left|\frac{\psi(x)-\psi(y)}{x-y}\right| \leq c \frac{\psi(y)}{y}
\end{aligned}
$$

5) if $k(x) \in V_{\lambda}$, then

$$
k(x) \leq c\left(\frac{y}{x}\right)^{\lambda} k(y), \quad x \leq y,
$$

and there exists $\varepsilon>0$ such that

$$
k(x) \leq c\left(\frac{y}{x}\right)^{\lambda-\varepsilon} k(y), \quad x \geq y ;
$$


6) if $\lambda \leq 1$, then

$$
\left|x^{\lambda}-y^{\lambda}\right| \leq c(x-y) y^{\lambda-1}, \quad x \geq y>0,
$$

and if $\lambda \geq 0$, then

$$
\left|x^{\lambda}-y^{\lambda}\right| \leq c(x-y) x^{\lambda-1}, \quad x \geq y>0 .
$$

Lemma 1. Let $k(x) \in V_{\lambda}, \lambda>0$ and let $\omega(x) \geq 0$ be an almost increasing function. Then $\omega(x) k(x) \leq c \int_{x}^{l} \frac{\omega(t) k(t)}{t} d t$ for $0<x<l / 2$.

Proof. By (2.8) we have

$$
\int_{x}^{l} \frac{\omega(t) k(t)}{t} d t \geq c \omega(x) x^{\lambda} k(x) \int_{x}^{l} \frac{d t}{t^{1+\lambda}} \geq c \omega(x) x^{\lambda} k(x) \int_{x}^{2 x} \frac{d t}{t^{1+\lambda}}=c \omega(x) k(x) .
$$

3. Mapping Properties of Convolution Operators in the Space

$$
H_{0}^{\omega}(\rho)
$$

The following theorem provides a Zygmund type estimate for the integral (1.1).

Theorem 1. Let $k(x) \in V_{\lambda}, 0<\lambda<1$ and $\varphi(x) \in C([a, b]), \varphi(a)=0$. Then

$$
\omega(K \varphi, h) \leq \operatorname{chk}(h) \omega(\varphi, h)+\operatorname{ch} \int_{h}^{b-a} \frac{k(t) \omega(\varphi, t)}{t} d t .
$$

Proof. Let $a=0$ for simplicity. We denote $g(x)=\varphi(x)-\varphi(0)$ and $f(x)=$ $\int_{0}^{x} k(x-t) g(t) d t$. For all $x, x+h \in[0, b]$ we have

$$
\begin{gathered}
f(x+h)-f(x)=\int_{-h}^{x}[g(x-t)-g(x)] k(t+h) d t- \\
-\int_{0}^{x}[g(x-t)-g(x)] k(t) d t+g(x)\left[\int_{-h}^{x} k(t+h) d t-\int_{0}^{x} k(t) d t\right] .
\end{gathered}
$$

So,

$$
\begin{gathered}
|f(x+h)-f(x)| \leq\left|\int_{-h}^{0}[g(x-t)-g(x)] k(t+h) d t\right|+ \\
+\left|\int_{0}^{x}[g(x-t)-g(x)][k(t)-k(t+h)] d t\right|+\left|g(x) \int_{x}^{x+h} k(t) d t\right|=A_{1}+A_{2}+A_{3} .
\end{gathered}
$$


Taking (2.8) and increasing of $\omega(\varphi, t)$ into account, we have for $A_{1}$ :

$$
A_{1} \leq \int_{0}^{h} \omega(\varphi, t) k(h-t) d t \leq c \omega(\varphi, h) k(h) \int_{0}^{h}\left(\frac{h}{h-t}\right)^{\lambda} d t \leq \operatorname{chk}(h) \omega(h) .
$$

For $A_{2}$, applying (2.2) and (2.9), we obtain in the case $h \geq x$ :

$$
\begin{aligned}
A_{2} & \leq \operatorname{ch} \int_{0}^{x} \frac{\omega(\varphi, t) k(t+h)}{t+h} d t \leq \operatorname{chk}(h) h^{\lambda-\varepsilon} \int_{0}^{x} \frac{\omega(\varphi, t) d t}{(t+h)^{1+\lambda-\varepsilon}}= \\
& =\operatorname{chk}(h) \int_{0}^{x / h} \frac{\omega(\varphi, h t) d t}{(t+1)^{1+\lambda-\varepsilon}} \leq \operatorname{chk}(h) \omega(\varphi, h) \int_{0}^{1} \frac{d t}{(t+1)^{1+\lambda-\varepsilon}} \leq \\
& \leq \operatorname{chk}(h) \omega(\varphi, h) .
\end{aligned}
$$

In the case $h<x$ we write $A_{2} \leq \int_{0}^{h}+\int_{h}^{x}=B_{1}+B_{2}$. For $B_{1}$ the estimate (3.3) is valid, while for $B_{2}$ we have

$$
B_{2} \leq c h \int_{h}^{b} \frac{\omega(\varphi, t) k(t)}{t} d t
$$

As regards $A_{3}$, we have in the case $h \geq x$ :

$$
\begin{aligned}
A_{3} & \leq c \omega(\varphi, h) k(x+h)(x+h)^{\lambda} \int_{x}^{x+h} \frac{d t}{t^{\lambda}} \leq \\
& \leq c \omega(\varphi, h) k(h) h^{\lambda} \int_{0}^{2 h} \frac{d t}{t^{\lambda}} \leq c \omega(\varphi, h) h k(h) .
\end{aligned}
$$

If $h<x$, we use Lemma 1 to obtain

$$
A_{3} \leq c \omega(\varphi, x) h k(x) \leq \operatorname{ch} \int_{x}^{b} \frac{\omega(\varphi, t) k(t) d t}{t} \leq c h \int_{h}^{b} \frac{\omega(\varphi, t) k(t)}{t} d t .
$$

Gathering all the estimates for $A_{i}, i=1,2,3$, we arrive at (3.1).

Theorem 2. Let $k(x) \in V_{\lambda}, 0<\lambda<1, \rho(x)=\psi(x-a), \psi(x) \in W_{\mu}$, $0<\mu<2$. Assume that

i) $\rho(x) \varphi(x) \in C([a, b])$ and $\left.\rho(x) \varphi(x)\right|_{x=a}=0$; 
ii) $\int_{0}^{b-a} t^{-\gamma} \omega(\rho \varphi, t) d t<\infty, \quad \gamma=\max (1, \mu)$. Then the following Zygmund type estimate holds:

$$
\omega(\rho K \varphi, h) \leq c h^{\gamma} k(h) \int_{0}^{h} \frac{\omega(\rho \varphi, t)}{t^{\gamma}} d t+c h \int_{h}^{b-a} \frac{\omega(\rho \varphi, t) k(t)}{t} d t,
$$

if $0<\mu<1+\lambda$ and

$$
\omega(\rho K \varphi, h) \leq c h \int_{0}^{h} \frac{\omega(\rho \varphi, t)}{t^{\mu}} d t+h \int_{h}^{b-a} \frac{\omega(\rho \varphi, t)}{t} d t,
$$

if $1+\lambda \leq \mu<2$.

Proof. Let $\varphi_{0}(x)=\rho(x) \varphi(x)$ and $a=0$ for simplicity. We have

$$
\begin{aligned}
\rho(x)(K \varphi)(x) & =\int_{0}^{x} k(x-t) \varphi_{0}(t) d t+\int_{0}^{x} \frac{\psi(x)-\psi(t)}{\psi(t)} k(x-t) \varphi_{0}(t) d t= \\
& =f_{1}(x)+f_{2}(x) .
\end{aligned}
$$

Since $\varphi_{0} \in C([0, b])$ and $\varphi_{0}(0)=0$, the first term $f_{1}(t)$ is covered by Theorem 1. To estimate $\omega\left(f_{2}, h\right)$ we represent the difference $f_{2}(x+h)-f_{2}(x)$ as

$$
\begin{aligned}
& f_{2}(x+h)-f_{2}(x)=\int_{x}^{x+h} \frac{\psi(x+h)-\psi(t)}{\psi(t)} \varphi_{0}(t) k(x+h-t) d t+ \\
& \quad+\int_{0}^{x} \frac{\psi(x+h)-\psi(x)}{\psi(t)} \varphi_{0}(t) k(x+h-t) d t+ \\
& +\int_{0}^{x} \frac{\psi(x)-\psi(t)}{\psi(t)}[k(x-t+h)-k(x-t)] \varphi_{0}(t) d t=I_{1}+I_{2}+I_{3} .
\end{aligned}
$$

Estimate for $I_{1}$. A) Let $0<\mu<1$ at first. Taking (2.4), (2.7) and (2.8) into account, we have

$$
\begin{gathered}
\left|I_{1}\right| \leq \int_{x}^{x+h} \frac{(x+h-t) k(x+h-t) \omega\left(\varphi_{0}, t\right) d t}{t} \leq \\
\leq \operatorname{chk}(h) \int_{x}^{x+h} \frac{\omega\left(\varphi_{0}, t-x\right) d t}{t-x} \leq \operatorname{chk}(h) \int_{0}^{h} \frac{\omega\left(\varphi_{0}, t\right) d t}{t} .
\end{gathered}
$$


B) If $1 \leq \mu<2$, then by (2.4),(2.6) and (2.8) we obtain

$$
\begin{gathered}
\left|I_{1}\right| \leq c \int_{0}^{h} \frac{(h-t) k(h-t)(x+h)^{\mu-1}}{(x+t)^{\mu-1}} \frac{\omega\left(\varphi_{0}, t\right)}{x+t} d t \leq \\
\quad \leq \operatorname{chk}(h) \int_{0}^{h} \frac{(x+h)^{\mu-1}}{t(x+h)^{\mu-1}} \omega\left(\varphi_{0}, t\right) d t .
\end{gathered}
$$

In the case $h<x$ we derive from (3.10)

$$
\left|I_{1}\right| \leq \operatorname{chk}(h) \int_{0}^{h} \frac{x^{\mu-1} \omega\left(\varphi_{0}, t\right) d t}{t(x+t)^{\mu-1}} \leq \operatorname{chk}(h) \int_{0}^{h} \frac{\omega\left(\varphi_{0}, t\right) d t}{t} .
$$

In the case $h>x$ the inequality (3.10) yields

$$
\left|I_{1}\right| \leq \operatorname{chk}(h) h^{\mu-1} \int_{0}^{h} \frac{\omega\left(\varphi_{0}, t\right) d t}{t(x+t)^{\mu-1}} \leq \operatorname{ch}^{\mu} k(h) \int_{0}^{h} \frac{\omega\left(\varphi_{0}, t\right) d t}{t^{\mu}} .
$$

So from (3.9), (3.11) and (3.12) there follows the estimate

$$
\left|I_{1}\right| \leq \operatorname{ch}^{\gamma} k(h) \int_{0}^{h} \frac{\omega\left(\varphi_{0}, t\right) d t}{t^{\gamma}}, \quad \gamma=\max (1, \mu) .
$$

Estimate for $I_{2}$. A) Let $0<\mu<1$. By (2.6) and (2.7) we have

$$
\left|I_{2}\right| \leq \operatorname{ch} \int_{0}^{x} \frac{k(x+h-t) \omega\left(\varphi_{0}, t\right) d t}{t} .
$$

In the case $h<x$ we represent (3.14) as $\left|I_{2}\right| \leq \int_{0}^{h}+\int_{h}^{(x+h) / 2}+\int_{(x+h) / 2}^{x}=$ $I_{2}^{\prime}+I_{2}^{\prime \prime}+I_{2}^{\prime \prime \prime}$. It is clear that

$$
I_{2}^{\prime} \leq \operatorname{chk}(h) \int_{0}^{h} \frac{\omega\left(\varphi_{0}, t\right) d t}{t} .
$$

Since $x+h-t \geq t$ in $I_{2}^{\prime \prime}$, we obtain

$$
I_{2}^{\prime \prime} \leq c h \int_{h}^{b} \frac{k(t) \omega\left(\varphi_{0}, t\right) d t}{t} .
$$


Further, $x+h-t \leq t$ in $I_{2}^{\prime \prime \prime}$, so by $(2.4)$

$$
\begin{aligned}
I^{\prime \prime \prime} & \leq \operatorname{ch} \int_{(x+h-t) / 2}^{x} \frac{k(x+h-t) \omega\left(\varphi_{0}, x+h-t\right) d t}{x+h-t} \leq \\
& \leq \operatorname{ch} \int_{h}^{b} \frac{\omega\left(\varphi_{0}, t\right) k(t) d t}{t} .
\end{aligned}
$$

If $h \geq x$, then (3.14) immediately yields

$$
\left|I_{2}\right| \leq \operatorname{chk}(h) \int_{0}^{h} \frac{\omega\left(\varphi_{0}, t\right) d t}{t} .
$$

B) Let now $1 \leq \mu<2$. Taking (2.1) into account, we have

$$
\left|I_{2}\right| \leq \operatorname{ch} \int_{0}^{x} \frac{(x+h)^{\mu-1} k(x+h-t) \omega\left(\varphi_{0}, t\right) d t}{t^{\mu}} .
$$

Hence

$$
\left|I_{2}\right| \leq c h^{\mu} k(h) \int_{0}^{h} \frac{\omega\left(\varphi_{0}, t\right) d t}{t^{\mu}}
$$

in the case $h \geq x$. If $h<x$, we represent (3.19) as

$$
\left|I_{2}\right| \leq \int_{0}^{h}+\int_{h}^{(x+h) / 2}+\int_{(x+h) / 2}^{x}=B_{1}+B_{2}+B_{3} .
$$

Taking into account that $x+h \leq 2(x+h-t)$ in $B_{1}$, in the case $0<\mu-1<\lambda$ we obtain

$$
\begin{aligned}
\left|B_{1}\right| & \leq \operatorname{ch} \int_{0}^{h} \frac{(x+h-t)^{\mu-1} k(x+h-t) \omega\left(\varphi_{0}, t\right) d t}{t^{\mu}} \leq \\
& \leq \operatorname{ch}^{\mu} k(h) \int_{0}^{h} \frac{\omega\left(\varphi_{0}, t\right) d t}{t^{\mu}}
\end{aligned}
$$

by (2.9). If $\mu-1>\lambda$, the function $t^{\mu-1} k(t)$ is bounded. So

$$
B_{1} \leq \operatorname{ch} \int_{0}^{h} \frac{\omega\left(\varphi_{0}, t\right) d t}{t^{\mu}} .
$$


Since $x+h \leq 2(x+h-t)$ again, we have

$$
B_{2} \leq \operatorname{ch} \int_{h}^{(x+h) / 2} \frac{(x+h-t)^{\mu-1} k(x+h-t)}{t^{\mu}} d t .
$$

Here $x+h-t \geq t$, so that

$$
B_{2} \leq \operatorname{ch} \int_{h}^{b} \frac{k(t) \omega\left(\varphi_{0}, t\right) d t}{t}
$$

by (2.9), if $\mu-1<\lambda$. If $\mu-1 \geq \lambda$, by boundedness of $t^{\mu-1} k(t)$ we have

$$
B_{2} \leq \operatorname{ch} \int_{h}^{b} \frac{\omega\left(\varphi_{0}, t\right) d t}{t^{\mu}} .
$$

To estimate $B_{3}$ we notice that $t \geq x+h-t$ in $B_{3}$. So by (2.4) we have

$$
B_{3} \leq c h \int_{x+h-t / 2}^{x} \frac{k(x+h-t) \omega\left(\varphi_{0}, x+h-t\right) d t}{x+h-t} \leq c h \int_{h}^{b} \frac{k(t) \omega\left(\varphi_{0}, t\right) d t}{t}
$$

Thus, $I_{2}$ admits the estimate

$$
\left|I_{2}\right| \leq c h^{\gamma} k(h)=\int_{0}^{h} \frac{\omega\left(\varphi_{0}, t\right) d t}{t^{\mu}}+c h \int_{h}^{b} \frac{k(t) \omega\left(\varphi_{0}, t\right) d t}{t}, \gamma=\max (1, \mu),
$$

if $\mu<1+\lambda$ and

$$
\left|I_{2}\right| \leq \operatorname{ch} \int_{0}^{h} \frac{\omega\left(\varphi_{0}, t\right) d t}{t^{\mu}}+\operatorname{ch} \int_{h}^{b} \frac{\omega\left(\varphi_{0}, t\right) d t}{t^{\mu}}
$$

if $\mu \geq 1+\lambda$.

Estimate for $I_{3}$. Let $0<\mu<1$. By (2.2) and (2.4) we have

$$
\left|I_{3}\right| \leq \operatorname{ch} \int_{0}^{x} \frac{k(x+h-t) \omega\left(\varphi_{0}, t\right) d t}{t}
$$

which coincides with the estimate in (3.14). If $1 \leq \mu<2$, we derive from $(2.1),(2.2)$ and (2.6):

$$
\left|I_{3}\right| \leq \operatorname{ch} \int_{0}^{x} \frac{x^{\mu-1} k(x+h-t) \omega\left(\varphi_{0}, t\right) d t}{t^{\mu}} \leq \operatorname{ch} \int_{0}^{x} \frac{(x+h)^{\mu-1} k(x+h-t) \omega\left(\varphi_{0}, t\right) d t}{t^{\mu}} .
$$


The latter coincides with (3.19). Gathering estimates for $I_{1}, I_{2}$ and $I_{3}$, we obtain (3.7)-(3.8).

Theorem 3. Let $\rho(x)=\psi(x-a), \psi(x) \in W_{\mu}, 0<\mu<2, k(t) \in V_{\lambda}$, $0<\lambda<1$. Assume that

i) $\mu<\lambda+1$;

ii) $t^{-\max (0, \mu-1)} \omega(t) \in Z, \quad t k(t) \omega(t) \in Z_{1}$.

Then the operator $K$ is bounded from $H_{0}^{\omega}(\rho)$ into $H_{0}^{\omega_{k}}(\rho)$ with $\omega_{k}(h)=$ $h k(h) \omega(h)$.

Proof. Let $f=K \varphi$ with $\varphi \in H_{0}^{\omega}(\rho)$ and let $a=0$. To prove that $f \in$ $H_{0}^{\omega_{k}}(\rho)$ we remark at first that

$$
\int_{0}^{b} \frac{\omega(\rho \varphi, t)}{t^{\gamma}} d t<\infty, \quad \gamma=\max (1, \mu)
$$

Therefore Zygmund type estimate (3.27) concerning the case $0<\mu<1+\lambda$ holds which gives

$$
\frac{\omega(\rho f, h)}{h k(h) \omega(h)} \leq\|\rho \varphi\|_{H_{0}^{\omega}}\left\{\frac{h^{\gamma-1}}{\omega(h)} \int_{0}^{h} \frac{\omega(t)}{t^{\gamma}} d t+\frac{1}{k(h) \omega(h)} \int_{h}^{b} \frac{\omega(t) k(t) d t}{t}\right\} .
$$

Hence by the condition ii) we have

$$
\frac{\omega(\rho f, h)}{h k(h) \omega(h)} \leq c\|\varphi\|_{H_{0}^{\omega}(\rho)} .
$$

It remains to prove that $\left.\rho(x) f(x)\right|_{x=0}=0$. After the change of variable $t=x-\xi x$ we have

$$
|\rho(x) f(x)| \leq x \psi(x) \int_{0}^{1} \frac{\left|\varphi_{0}(x-x \xi)\right| k(x \xi) d \xi}{\psi(x-x \xi)} .
$$

Since $\varphi_{0}(0)=0$, this yields

$$
|\rho(x) f(x)| \leq c x \psi(x) \int_{0}^{1} \frac{\omega\left(\varphi_{0}(, 1-\xi) d \xi\right.}{\psi(x-x \xi)} .
$$

According to (2.6) and (2.8) we see that

$$
|\rho(x) f(x)| \leq \operatorname{cxk}(x) \int_{0}^{1} \frac{\omega\left(\varphi_{0}, 1-\xi\right) d \xi}{\xi^{\lambda}(1-\xi)^{\gamma}}=c_{1} x k(x) \rightarrow 0
$$

as $x \rightarrow 0$ in view of (3.27). So $\left.\rho(x) f(x)\right|_{x=0}=0$. 
Corollary 1. The operator (1.1) with the kernel $k(t)=t^{\alpha-1}\left(\ln \frac{\gamma}{t}\right)^{\beta}, \gamma>$ $b-a, 0<\alpha<1, \beta \geq 0$ is bounded from $H_{0}^{\omega}(\rho)$ into $H_{0}^{\omega_{\alpha, \beta}}(\rho)$, where $\rho(x)=$ $\psi(x-a), \psi(x) \in W_{\mu}$ and $\omega \alpha, \beta(h)=\omega(h) h^{\alpha}\left(\ln \frac{\gamma}{h}\right)^{\beta}$ under the assumption that $0<\mu<2-\alpha$ and $h^{-\max (0, \mu-1)} \omega(h) \in Z, h^{\alpha}\left(\ln \frac{\gamma}{h}\right)^{\beta} \omega(h) \in Z_{1}$.

In the case $\psi(x)=x^{\mu}$ and $\omega(h)=h^{\lambda}$ the assertion of Corollary 1 was proved in [6] (see [17], Theorem 21.2).

Corollary 2. The operator of the form $\int_{x}^{b} k(t-x) \varphi(t) d t$ is bounded from $H_{0}^{\omega}(\rho)$ into $H_{0}^{\omega_{k}}(\rho)$ under the assumptions of Theorem 3 if the requirement $\left.\rho f\right|_{x=a}=0$ in the definition of the space $H_{0}^{\omega}(\rho)$ is replaced by $\left.\rho f\right|_{x=b}=0$.

4. Mapping Properties of Fractional Differentiation in the

$$
\text { SPACES } H_{0}^{\omega}(\rho)
$$

Now we give Zygmund type estimate for the fractional derivative (1.5).

Theorem 4. Let $\rho(x)=\psi(x-a), \psi(x) \in W_{\mu}, 0<\mu<2$, and

$$
\int_{0}^{b-a} \frac{\omega(\rho f, t)}{t^{\alpha+\mu}} d t<\infty, \quad \gamma=\max (1, \mu) .
$$

Then

$$
\omega\left(\rho D_{a+}^{\alpha} f, h\right) \leq c h^{\gamma-1} \int_{0}^{h} \frac{\omega(\rho f, t)}{t^{\alpha+\gamma}} d t .
$$

Proof. According to (1.5) we have

$$
\begin{aligned}
& \rho(x)\left(D_{a+}^{\alpha} f\right)(x)=\frac{\rho(x) f(x)}{\Gamma(1-\alpha)(x-a)^{\alpha}}+ \\
& +\frac{\alpha}{\Gamma(1-\alpha)} \rho(x) \int_{0}^{x-a} \frac{f(x)-f(x-t)}{t^{1+\alpha}} d t .
\end{aligned}
$$

We set $a=0$ and denote $\theta(x)=\psi(x) \int_{0}^{x} \frac{f(x)-f(x-t)}{t^{1+\alpha}} d t$. To estimate the difference $\theta(x+h)-\theta(x)$ we represent it in the form $\theta(x+h)-\theta(x)=$ $\sum_{k=1}^{8} A_{k}(x)$ (as in [13] in the purely power case), where

$$
\begin{aligned}
& A_{1}(x)=\left[1-\frac{\psi(x)}{\psi(x+h)}\right] \int_{0}^{x} \frac{g(x+h)-g(y)}{(x+h-y)^{1+\alpha}} d y \\
& A_{2}(x)=[\psi(x+h)-\psi(x)] \int_{0}^{x+h} \frac{g(y)}{(x+h-y)^{1+\alpha}}\left[\frac{1}{\psi(x+h)}-\frac{1}{\psi(y)}\right] d y,
\end{aligned}
$$




$$
\begin{aligned}
& A_{3}(x)=\frac{\psi(x)}{\psi(x+h)} \int_{x}^{x+h} \frac{g(x+h)-g(y)}{(x+h-y)^{1+\alpha}} d y, \\
& A_{4}(x)=\psi(x) \int_{x}^{x+h} \frac{g(y)}{(x+h-y)^{1+\alpha}}\left[\frac{1}{\psi(x+h)}-\frac{1}{\psi(y)}\right] d y, \\
& A_{5}(x)=\int_{0}^{x}[g(x)-g(y)]\left[(x+h-y)^{-1-\alpha}-(x-y)^{-1-\alpha}\right] d y, \\
& A_{6}(x)=\psi(x) \int_{0}^{x} g(y)\left[\frac{1}{\psi(x)}-\frac{1}{\psi(y)}\right]\left[(x+h-y)^{-1-\alpha}-(x-y)^{-1-\alpha}\right] d y, \\
& A_{7}(x)=\frac{1}{\alpha} \frac{\psi(x)}{\psi(x+h)}[g(x+h)-g(x)]\left[h^{-\alpha}-(x+h)^{-\alpha}\right], \\
& A_{8}(x)=\frac{1}{\alpha} \psi(x) g(x)\left[\frac{1}{\psi(x+h)}-\frac{1}{\psi(x)}\right]\left[h^{-\alpha}-(x+h)^{-\alpha}\right] .
\end{aligned}
$$

Estimate for $A_{1}$. By (2.1) we have

$$
\left|A_{1}\right| \leq c \frac{h}{x+h} \int_{0}^{x+h} \frac{\omega(g, x+h-y)}{(x+h-y)^{1+\alpha}} d y=c \frac{h}{x+h} \int_{0}^{x+h} \frac{\omega(g, t)}{t^{1+\alpha}} d t .
$$

If $h \geq x$, it is obvious that

$$
\left|A_{1}\right| \leq c \int_{0}^{h} \frac{\omega(g, t)}{t^{1+\alpha}} d t
$$

If $h<x$ represent (4.3) as $\left|A_{1}\right| \leq \int_{0}^{h}+\int_{h}^{x+h}=A_{1}^{\prime}+A_{1}^{\prime \prime}$. For $A_{1}^{\prime}$ the estimate (4.4) holds. As regards $A_{1}^{\prime \prime}$, applying (2.4) and (2.5), we have

$$
\begin{aligned}
A_{1}^{\prime \prime} & \leq c h \int_{x}^{x+h} \frac{\omega(g, t)}{t} \frac{d t}{t^{1+\alpha}} \leq c \omega(g, h) \int_{h}^{\infty} \frac{d t}{t^{1+\alpha}} \leq \\
& \leq c \frac{\omega(g, h)}{h^{\alpha}} \leq c \int_{0}^{h} \frac{\omega(g, t)}{t^{1+\alpha}} d t .
\end{aligned}
$$


Estimate for $A_{2}$. A) In the case $0<\mu<1$, using (2.7), we obtain

$$
\left|A_{2}\right| \leq c \frac{h}{x+h} \int_{0}^{x+h} \frac{\omega(g, y)}{y(x+h-y)^{\alpha}} d y=\int_{0}^{h / 2}+\int_{h / 2}^{x+h}=A_{2}^{\prime}+A_{2}^{\prime \prime} .
$$

Obviously, $A_{2}^{\prime} \leq c \int_{0}^{h} \frac{\omega(g, t)}{t^{1+\alpha}} d t$. Using (2.4) and (2.5), we derive the following estimate for $A_{2}^{\prime \prime}$ :

$$
A_{2}^{\prime \prime} \leq c \frac{\omega(g, h)}{x+h} \int_{0}^{x+h} \frac{d y}{(x+h-y)^{\alpha}}=c_{1} \frac{\omega(g, h)}{(x+h)^{\alpha}} \leq c_{2} \int_{0}^{h} \frac{\omega(g, t)}{t^{1+\alpha}} d t .
$$

B) If $1 \leq \mu<2-\alpha$, taking (2.1) and (2.6) into account, we obtain for $A_{2}$ :

$$
\left|A_{2}\right| \leq c \frac{h}{(x+h)^{2-\mu}} \int_{0}^{h} \frac{\omega(g, y) d y}{y^{\mu}(x+h-y)^{\alpha}}=\int_{0}^{h / 2}+\int_{h / 2}^{x+h}=B_{1}+B_{2} .
$$

It is obvious that

$$
B_{1} \leq \operatorname{ch}^{\mu-1} \int_{0}^{h} \frac{\omega(g, t)}{t^{\mu+\alpha}} d t .
$$

As regards $B_{2}$, we apply (2.4) and obtain

$$
\begin{aligned}
B_{2} & \leq c \frac{\omega(g, h)}{(x+h)^{2-\mu}} \int_{h / 2}^{x+h} \frac{d y}{y^{\mu-1}(x+h-y)^{\alpha}} \leq \\
& \leq c \frac{\omega(g, h)}{(x+h)^{\alpha}} \int_{0}^{1} \frac{d t}{t^{\mu-1}(1-t)^{\alpha}}=c_{1} \frac{\omega(g, h)}{(x+h)^{\alpha}} .
\end{aligned}
$$

Using then (2.5), we notice that the estimate for $B_{2}$ is the same as in (4.6). Estimate for $A_{3}$. Since $\psi(x)$ is almost increasing, we have

$$
\left|A_{3}\right| \leq c \int_{x}^{x+h} \frac{\omega(g, x+h-y)}{(x+h-y)^{1+\alpha}} d y=c \int_{0}^{h} \frac{\omega(g, t)}{t^{1+\alpha}} d t .
$$

Estimate for $A_{4}$. Let $0<\mu<1$ at first. In view of (2.7) we have

$$
\left|A_{4}\right| \leq c \int_{0}^{h} \frac{\omega(g, x+h-t)}{t^{\alpha}(x+h-t)} d t
$$


In the case $h<x$ we have $t \leq x+h-t$ in (4.7). So by (2.4) we obtain $\left|A_{4}\right| \leq$ $c \int_{0}^{h} \frac{\omega(g, t)}{t^{1+\alpha}} d t$. If $h \geq x$, we represent (4.7) as $\left|A_{4}\right| \leq \int_{0}^{(x+h) / 2}+\int_{(x+h) / 2}^{h}$. Since $t \leq x+h-t$ and $t \geq x+h-t$ in the first and second terms, respectively, by (2.4) we derive that

$$
\left|A_{4}\right| \leq c \int_{0}^{(x+h) / 2} \frac{\omega(g, t)}{t^{1+\alpha}} d t+c \int_{(x+h) / 2}^{h} \frac{\omega(g, x+h-t)}{(x+h-t)^{1+\alpha}} d t \leq c \int_{0}^{h} \frac{\omega(g, t)}{t^{1+\alpha}} d t .
$$

Let now $1 \leq \mu<2-\alpha$. Using (2.1) and (2.6), we get

$$
\left|A_{4}\right| \leq \frac{c}{(x+h)^{1-\mu}} \int_{0}^{h} \frac{\omega(g, x+h-t)}{(x+h-t)^{\mu} t^{\alpha}} d t .
$$

If $h<x$, by (2.4) we have

$$
\left|A_{4}\right| \leq c(x+h)^{\mu-1} \int_{0}^{h} \frac{\omega(g, t) d t}{(x+h-t)^{\mu-1} t^{1+\alpha}} \leq c \int_{0}^{h} \frac{\omega(g, t)}{t^{1+\alpha}} d t .
$$

If $h \geq x$, then $\left|A_{4}\right| \leq \int_{0}^{(x+h) / 2}+\int_{(x+h) / 2}^{h}$. We use (2.4) in the first term and the inequality $t^{\alpha} \geq(x+h-t)^{\alpha}$ in the second. This yields

$$
\begin{aligned}
& \left|A_{4}\right| \leq \int_{0}^{(x+h) / 2} \frac{\omega(g, t)}{t^{1+\alpha}} d t c(x+h)^{\mu-1} \int_{(x+h) / 2}^{h} \frac{\omega(g, x+h-t)}{(x+h-t)^{\alpha+\mu}} d t \leq \\
& \leq c \int_{0}^{h} \frac{\omega(g, t)}{t^{1+\alpha}} d t+c h^{\mu-1} \int_{x}^{(x+h) / 2} \frac{\omega(g, t)}{t^{\alpha+\mu}} d t \leq c h^{\mu-1} \int_{0}^{h} \frac{\omega(g, t)}{t^{\alpha+\mu}} d t .
\end{aligned}
$$

Estimate for $A_{5}$. Applying (2.11), we have

$$
\left|A_{5}\right| \leq \operatorname{ch} \int_{0}^{h} \omega(g, x-y) \frac{d y}{(x+h-y)(x-y)^{1+\alpha}}=\operatorname{ch} \int_{0}^{x} \frac{\omega(g, t) d t}{t^{1+\alpha}(t+h)} .
$$

In the case $h \geq x$ it is clear that

$$
\left|A_{5}\right| \leq c \int_{0}^{h} \frac{\omega(g, t)}{t^{1+\alpha}} d t
$$


If $h<x$, then $\left|A_{5}\right| \leq \int_{0}^{h}+\int_{h}^{x}=A_{5}^{\prime}+A_{5}^{\prime \prime}$ with the same estimate as in (4.8) for $A_{5}^{\prime}$. As regards $A_{5}^{\prime \prime}$, we have

$$
A_{5}^{\prime \prime} \leq c \omega(g, h) \int_{h}^{\infty} \frac{d t}{t^{1+\alpha}} \leq c \frac{\omega(g, h)}{h^{\alpha}} \leq c \int_{0}^{h} \frac{\omega(g, t)}{t^{1+\alpha}} d t
$$

by (2.4) and (2.5).

Estimate for $A_{6}$. A) Let $0<\mu<1$ at first. Applying (2.7) and (2.11) we arrive at

$$
\left|A_{6}\right| \leq \operatorname{ch} \int_{0}^{x} \frac{\omega(g, y) d y}{y(x+h-y)(x-y)^{\alpha}}=\int_{0}^{x / 2}+\int_{x / 2}^{x}=A_{6}^{\prime}+A_{6}^{\prime \prime} .
$$

For $A_{6}^{\prime}$ we have

$$
A_{6}^{\prime} \leq \operatorname{ch} \int_{0}^{x / 2} \frac{\omega(g, y) d y}{(y+h) y^{1+\alpha}}
$$

If $h<x, A_{6}^{\prime} \leq \int_{0}^{h / 2}+\int_{h / 2}^{x / 2}=K_{1}+K_{2}$. It is evident that $K_{1}$ admits the same estimate as in (4.9). For $K_{2}$ the application of (2.5) provides the same result: $K_{2} \leq c \omega(g, h) \int_{h / 2}^{\infty} \frac{d t}{t^{1+\alpha}} \leq c \int_{0}^{h} \frac{\omega(g, t)}{t^{1+\alpha}} d t$. If $h \geq x$, then immediately

$$
A_{6}^{\prime} \leq c \int_{0}^{h} \frac{\omega(g, t)}{t^{1+\alpha}} d t
$$

To estimate $A_{6}^{\prime}$ we remark that $y \geq x-y$ so that

$$
A_{6}^{\prime \prime} \leq \operatorname{ch} \int_{x / 2}^{x} \frac{\omega(g, x-y) d y}{(x-y)^{1+\alpha}(x+h-y)}=c h \int_{0}^{x / 2} \frac{\omega(g, t) d t}{t^{1+\alpha}(t+h)},
$$

which is the same as in (4.10) and so $A_{6}^{\prime \prime}$ admits the same estimates as in (4.5).

B) Let $1 \leq \mu<2-\alpha$. Using (2.1), (2.6) and (2.11), we have

$$
A_{6} \leq \operatorname{ch} x^{\mu-1} \int_{0}^{x} \frac{\omega(g, y) d y}{y^{\mu}(x+h-y)(x-y)^{\alpha}}=\int_{0}^{x / 2}+\int_{x / 2}^{x}=U_{1}+U_{2} .
$$


If $h<x$, we set $U_{1}=\int_{0}^{h / 2}+\int_{h / 2}^{x / 2}=U_{1}^{\prime}+U_{1}^{\prime \prime}$, whence easy calculations yield the inequality

$$
U_{1} \leq \operatorname{ch} \int_{0}^{h} \frac{\omega(g, t) d t}{t^{1+\alpha}}+c h^{\mu-1} \int_{0}^{h} \frac{\omega(g, t) d t}{t^{\mu+\alpha}} \leq c h^{\mu-1} \int_{0}^{h} \frac{\omega(g, t) d t}{t^{\mu+\alpha}} .
$$

For $U_{2}$ by (2.5) we have

$$
\begin{aligned}
U_{2} & \leq c h \int_{x / 2}^{x} \frac{\omega(g, y) d y}{y(x+h-y)(x-y)^{\alpha}} \leq c \omega(g, h) \int_{0}^{x / 2} \frac{d t}{t^{\alpha}(t+h)} \leq \\
& \leq c \frac{\omega(g, h)}{h^{\alpha}} \int_{0}^{\infty} \frac{d \xi}{\xi^{\alpha}(1+\xi)} \leq c \int_{0}^{h} \frac{\omega(g, t) d t}{t^{1+\alpha}} .
\end{aligned}
$$

If $h \geq x$, the estimation of $U_{1}$ and $U_{2}$ is easy and provides the same as in (4.2). Gathering all the estimates, we obtain $\left|A_{6}\right| \leq c h^{\gamma-1} \int_{0}^{h} \frac{\omega(g, t)}{t^{\alpha+\gamma}} d t$, $\gamma=\max (1, \mu)$.

Estimate for $A_{7}$. Applying (2.5) and (2.10) and almost increasing of $\psi(x)$, we easily obtain

$$
\left|A_{7}\right| \leq c \int_{0}^{h} \frac{\omega(g, t)}{t^{1+\alpha}} d t
$$

Estimate for $A_{8}$. Using the inequalities (2.1) and (2.10) for $0<\mu<2-\alpha$, we make sure of validity of the estimate (4.13) for $A_{8}$ as well.

It remains to consider the first term

$$
r(x)=\frac{\psi(x-a) f(x)}{\Gamma(1-\alpha)(x-a)^{\alpha}}=\frac{g(x)}{\Gamma(1-\alpha)(x-a)^{\alpha}}
$$

in (4.2). Since $g(x) \in H_{0}^{\omega}$, we have the estimate

$$
|r(x+h)-r(x)| \leq c \int_{0}^{h} \frac{\omega(g, t) d t}{t^{1+\alpha}},
$$

which is derived by direct calculations under the assumptions of the theorem.

Collecting all the estimates for $A_{i}, i=1, \ldots, 8$, and (4.14), we obtain the required inequality (4.1). 
Theorem 5. Let $\rho(x)=\psi(x-a), \psi(x) \in W_{\mu}, 0<\mu<2-\alpha$ and let

1) $\omega(t) \neq 0, \quad t>0$,

2) $\left.\omega(t) t^{-\alpha}\right|_{t=0}=0$,

3) $\omega(t) t^{1-\alpha-\gamma} \in Z, \quad \gamma=\max (1, \mu)$.

Then the operator $D_{a+}^{\alpha}$ continuously maps $H_{0}^{\omega}(\rho)$ into $H_{0}^{\omega-\alpha}(\rho)$ with $\omega_{-\alpha}(h)=h^{-\alpha} \omega(h)$.

Proof. Let $f(x) \in H_{0}^{\omega}(\rho)$ and $\varphi(x)=D_{a^{+}}^{\alpha} f(x)$. To show that

$$
\sup _{0<h \leq b-a} \frac{h^{\alpha} \omega(\rho \varphi, h)}{\omega(h)}=c<\infty
$$

we observe that the inclusion $\omega(t) t^{1-\alpha-\gamma} \in Z$ implies convergence of the integral $\int_{0}^{b-a} \omega(t) t^{-\alpha-\gamma} d t$, so Theorem 4 is applicable. Using the estimate (4.1) of Theorem 4, we obtain

$$
\frac{h^{\alpha} \omega(\rho \varphi, h)}{\omega(h)} \leq \frac{h^{\alpha+\gamma-1} \int_{0}^{1} \omega(\rho f, t) t^{-\alpha-\gamma} d t}{\omega(h)} \leq c\|f\|_{H_{0}^{\omega}(\rho)} .
$$

It remains to show that $\left.\rho(x) \varphi(x)\right|_{x=a}=0$. By (4.2) we have

$$
\begin{gathered}
|\rho(x) \varphi(x)| \leq \frac{\omega(\rho f, x-a)}{\Gamma(1-\alpha)(x-a)^{\alpha}}+\frac{\alpha}{\Gamma(1-\alpha)} \int_{0}^{x-a} \frac{\omega(\rho f, t) d t}{t^{1+\alpha}}+ \\
+\frac{\alpha}{\Gamma(1-\alpha)} \int_{0}^{x-a} \frac{|\psi(x-a)-\psi(x-a-t)| \omega(\rho f, x-a-t) d t}{t^{1+\alpha} \psi(x-a-t)}= \\
=D_{1}+D_{2}+D_{3} .
\end{gathered}
$$

Here $D_{1} \leq c\|f\| H_{0}^{\omega}(\rho) \frac{\omega(x-a)}{(x-a)^{\alpha}}$, the condition (4.16) implies $\left.\omega(x) x^{-\alpha}\right|_{x=0}=$ 0 . So $\lim _{x \rightarrow a} D_{1}=0$. The equality $\lim _{x \rightarrow a} D_{2}=0$ is obvious by the existence of the integral in $D_{2}$.

For the term $D_{3}$ in the case $0<\mu<1$ we have by (2.7)

$$
D_{3} \leq c \int_{0}^{x-a} \frac{\omega(\rho f, x-a-t) d t}{t^{\alpha}(x-a-t)} .
$$

We evaluate this separately for $x-a-t \geq t$ and $x-a-t \leq t$ by means of (2.4):

$$
D_{3} \leq c \int_{0}^{(x-a) / 2} \frac{\omega(\rho f, t) d t}{t^{1+\alpha}}+c \int_{(x-a) / 2}^{x-a} \frac{\omega(\rho f, x-a-t)}{(x-a-t)^{1+\alpha}} d t,
$$

whence $\lim _{x \rightarrow a} D_{3}=0$. 
If $1 \leq \mu \leq 2-\alpha$, we use (2.1) and (2.5) and obtain

$$
D_{3} \leq c \int_{0}^{x-a} \frac{\omega(\rho f, x-a-t)}{(x-a)^{1-\mu}(x-a-t)^{\mu} t^{\alpha}}=\int_{0}^{(x-a) / 2}+\int_{(x-a) / 2}^{x-a}
$$

and similar to what we did above we have

$$
\begin{aligned}
D_{3} & \leq c \int_{0}^{(x-a) / 2} \frac{(x-a)^{\mu-1} \omega(\rho f, t)}{t^{\alpha+\mu}} d t+c \int_{(x-a) / 2}^{x-a} \frac{(x-a)^{\mu-1} \omega(\rho f, x-a-t)}{(x-a-t)^{\alpha+\mu}} d t \leq \\
& \leq(x-a)^{\mu-1} \int_{0}^{(x-a) / 2} \frac{\omega(\rho f, t) d t}{t^{\alpha+\mu}},
\end{aligned}
$$

so that $\lim _{x \rightarrow a} D_{3}=0$. Therefore, $\lim _{x \rightarrow a} \rho(x) \varphi(x)=0$.

Corollary 1. Let $\varphi(x) \in W_{\mu} 0<\mu<2-\alpha$, and let $\omega(t)$ be an almost increasing function on $[0, b-a]$ such that

1) $\omega(0)=0, \omega(t) \neq 0$ for $t \in(0, b-a]$;

2) $\omega(t) t^{-1-\gamma} \in Z, \quad \gamma=\max (1, \mu)$.

Then the operator $D_{a^{+}}^{\alpha}$ of fractional differentiation continuously maps $H_{0}^{\omega_{\alpha}}(\rho)$ into $H_{0}^{\omega}$ with $\rho(x)=\varphi(x-a), \omega_{\alpha}(h)=h^{\alpha} \omega(h)$.

Another corollary (of Theorems 5 and 3 ) will be related to the following Love's index law [8]:

$$
I_{0+}^{\gamma} x^{\alpha} I_{0+}^{\beta} x^{\gamma} I_{0+}^{\alpha} x^{\beta} f(x)=f(x), \quad \alpha+\beta+\gamma=0
$$

well known in fractional calculus. This corollary will provide conditions guaranteeing validity of (4.18) for functions $f \in H_{0}^{\omega}(\rho)$. For simplicity we restrict ourselves with the cases $\omega(x)=x^{\lambda}$ and $\rho(x)=x^{\mu}$. The notation

$$
\alpha^{*}= \begin{cases}\alpha, & \alpha \leq 1 \\ 1, & \alpha \geq 1\end{cases}
$$

is used below.

Corollary 2. Relation (4.18) is valid for all functions $f(x) \in H_{0}^{\lambda}\left(x^{\mu}\right)$ and all $\alpha, \beta, \gamma \in R^{1}$ such that $\alpha+\beta+\gamma=0$, if the number $\lambda \in(0,1]$ satisfies the conditions $\lambda>-\alpha,(\lambda+\alpha)^{*}+\beta>0\left[(\lambda+\alpha)^{*}+\beta\right]^{*}+\gamma>0$ while the weight exponent $\mu$ satisfies the conditions

$$
\begin{array}{ll}
\text { 1) } & \mu<(\lambda+\alpha)^{*}+1 \\
\text { 2) } & \mu<\left[(\lambda+\alpha)^{*}+\beta\right]^{*}+1 \\
\text { 3) } & \mu<\left\{\left[(\lambda+\alpha)^{*}+\gamma\right\}^{*}+1\right.
\end{array}
$$




\section{A Theorem on Isomorphism}

In Theorem 3 and Corollary 1 of Theorem 5 it was proved that

$$
\begin{aligned}
& I_{a+}^{a}: H_{0}^{\omega}(\rho) \longrightarrow H_{0}^{\omega_{\alpha}}(\rho), \\
& D_{a+}^{a}: H_{0}^{\omega_{\alpha}}(\rho) \longrightarrow H_{0}^{\omega}(\rho)
\end{aligned}
$$

under the appropriate assumptions on $\omega(h)$ and $\rho(x)$. To derive the assertion $I_{a+}^{\alpha}\left[H_{0}^{\omega}(\rho)\right]=H_{0}^{\omega_{\alpha}}(\rho)$ it remains to show that any function in $H_{0}^{\omega_{\alpha}}(\rho)$ is representable by the fractional integral of a function in $H_{0}^{\omega}(\rho)$. This will be the goal of Theorem 6 below. Preliminarily we state two auxiliary assertions we need.

Lemma 2 ([17], p.185; p.231 in English ed.). In order a function $f(x)$ to be representable as $f=I_{a+}^{\alpha} \varphi, \varphi \in L_{p}(a, b),-\infty<a<b<\infty$, it is necessary and sufficient that

i) $f(x)(x-a)^{-\alpha} \in L_{p}(a, b)$;

ii) $\left\|\psi_{\varepsilon}\right\|_{L_{p}} \leq c<\infty$ with $c$ not depending on $\varepsilon$, where

$$
\psi_{\varepsilon}(x)=\int_{a}^{x-\varepsilon} \frac{f(x)-f(t)}{(x-t)^{1+\alpha}} d t,
$$

for $a+\varepsilon<x<b$ and $\psi_{\varepsilon}(x)=0$ for $a<x<a+\varepsilon$.

A close version of Lemma 2 can be found in [16]. (See also [3] for another version under additional assumptions that $f \in L_{p}$ and $1<p<1 / 2 \alpha$ ).

Lemma 3. Let $\omega(t) t^{-\delta} \in Z$. There exists $p>1$ such that $\omega(t) t^{-1-\delta} \in$ $L_{p}(0,1)$.

Proof. It is known that the inclusion $\varphi(t) \in Z$ implies existence of $\varepsilon \in(0,1)$ such that $t^{-\varepsilon} \varphi(t)$ is almost increasing (see, e.g. [1]). Therefore, there exists $\varepsilon \in(0,1)$ such that $t^{-\varepsilon-\delta} \omega(t)$ is bounded. So $\omega(t) \leq c t^{\delta+\varepsilon}$ and to have a finite $L_{p}$-norm for $\omega(t) / t^{1+\delta}$ we must choose $p<\frac{1}{1-\varepsilon}$.

Theorem 6 (On isomorphism). Let $\psi(x) \in W_{\mu}, 0<\mu<2-\alpha$ and let $\omega(t)$ be a continuous function such that $\omega(t) t^{1-\gamma} \in Z, \omega(t) t^{\alpha} \in Z_{1}$, $\gamma=\max (1, \mu), 0<\alpha<1$. Then the fractional integration operator $I_{a+}^{\alpha}$ isomorphically maps the weighted space $H_{0}^{\omega}(\rho)$ with $\rho(x)=\psi(x-a)$ onto the space $H_{0}^{\omega_{\alpha}}(\rho)$ with the same weight and the characteristic $\omega_{\alpha}(h)=h^{\alpha} \omega(h)$.

Proof. In view of (5.1)-(5.2) it is sufficient to prove the representability of a function $f \in H_{0}^{\omega_{\alpha}}(\rho)$ by a fractional integral. Aiming to apply Lemma 2 we shall prove that there exists $p>1$ such that conditions i)-ii) of Lemma 2 are satisfied. 
The estimate

$$
\frac{|f(x)|}{(x-a)^{\alpha}} \leq c\|f\|_{H_{0}^{\omega_{\alpha}}} \frac{\omega(x-a)}{(x-a)^{\mu}}
$$

is valid for any $f(x) \in H_{0}^{\omega_{\alpha}}(\rho)$. Really, by (2.6) we have

$$
\frac{|f(x)|}{(x-a)^{\alpha}} \leq c \frac{|\rho(x) f(x)|}{(x-a)^{\mu+\alpha}}
$$

which immediately provides (5.3).

Since $\omega(x) / x^{\delta-1} \in Z$, from (5.3) and Lemma 3 we conclude that there exists $p>1$ such that the condition i) of Lemma 2 is satisfied.

For this $p$ we shall show that a constant $c>0$ exists such that

$$
\left\|\psi_{\varepsilon}\right\|_{L_{p}} \leq c \leq \infty
$$

We set $g(x)=f(x) \psi(x-a)$ and have

$$
\left|\psi_{\varepsilon}(x)\right| \leq \frac{1}{\psi(x-a)} \int_{a}^{x-\varepsilon} \frac{|g(x)-g(t)|}{(x-t)^{1+\alpha}} d t+\int_{a}^{x-\varepsilon} \frac{\left|g(t)\left[\frac{1}{\psi(x-a)}-\frac{1}{\psi(t-a)}\right]\right|}{(x-t)^{1+\alpha}} d t .
$$

To estimate $F_{1}$ we use (2.6) and obtain

$$
F_{1} \leq \frac{c}{(x-a)^{\mu}} \int_{a}^{x} \frac{\omega(g, x-t)}{(x-t)^{1+\alpha}} d t \leq \frac{c}{(x-a)^{\gamma}} \int_{a}^{x} \frac{\omega(g, x-t)}{(x-t)^{1+\alpha}} d t
$$

Since $g(x) \in H_{0}^{\omega_{\alpha}}(\rho)$, it is easily proved that (5.6) yields

$$
F_{1} \leq \frac{c}{(x-a)^{\gamma}}
$$

For $F_{2}$ in the case $0<\mu<1$ we use (2.6) and (2.7) and obtain

$$
F_{2} \leq \frac{c}{(x-a)} \int_{0}^{x-a} \frac{\omega(g, x-t-a)}{t^{\alpha}(x-t-a)} d t=\int_{0}^{(x-a) / 2}+\int_{(x-a) / 2}^{x-a} .
$$

Hence after simple calculations

$$
\begin{aligned}
F_{2} & \leq \frac{c}{x-a} \int_{0}^{(x-a) / 2} \frac{\omega(t) d t}{t}+\frac{c}{x-a} \int_{(x-a) / 2}^{x-a} \frac{\omega(x-t-a) d t}{x-t-a} \leq \\
& \leq c \frac{\omega(x-a)}{x-a}+\frac{c}{x-a} \int_{0}^{x-a} \frac{\omega(t)}{t} d t .
\end{aligned}
$$


Since the condition $\omega(t) t^{1-\mu} \in Z$ with $0<\mu<1$ implies $\omega(t) \in Z$, we derive from (5.8) the estimate $F_{2} \leq c \omega(x-a) /(x-a)$.

Let now $1 \leq \mu<2-\alpha$. We use inequalities (2.1) and (2.6) to obtain

$$
\begin{gathered}
F_{3} \leq \frac{c}{x-a} \int_{0}^{x-a} \frac{\omega(g, x-t-a)}{t^{\alpha}(x-t-a)^{\mu}} d t \leq \frac{c}{x-a} \int_{0}^{x-a} \frac{\omega(x-t-a) d t}{t^{\alpha}(x-t-a)^{\mu-a}}= \\
=\int_{0}^{(x-a / 2}+\int_{(x-a) / 2}^{x-a} .
\end{gathered}
$$

Calculations and arguments similar to those in the case $0<\mu<1$ give the estimate $F_{2} \leq c \frac{\omega(x-a)}{(x-a)^{\mu}}$. Therefore,

$$
F_{2} \leq c \frac{\omega(x-a)}{(x-a)^{\gamma}}, \quad \gamma=\max (1, \mu)
$$

So, from (5.8), (5.9) we obtain $\left|\psi_{\varepsilon}(x)\right| \leq c \frac{\omega(x-a)}{(x-a)^{\gamma}} \in L_{p}$. Hence $\|\psi\|_{L_{p}} \leq c$.

\section{REFERENCES}

1. N.K. Bari and S.B. Stechkin, Best approximation and differential properties of two conjugate functions. (Russian) Trudy Mosk. Mat. Obshch. $\mathbf{5}(1956), 483-522$.

2. G.H. Hardy and J.E. Littlewood, Some properties of fractional integrals, I. Math. Z. 27(1928), 565-606.

3. D.L. Herson and P. Heywood, On the range of some fractional integrals. J. London Math. Soc. (2), 8(1974), 607-614.

4. N.K. Karapetyants, Kh.M. Murdaev and A.Ya. Yakubov, Zygmund type estimate for fractional integrodifferentiation in the spaces of functions defined by integral continuity moduli and isomorphism of generalized Hölder spaces. (Russian) Rostov on Don. Deposited in VINITI (Moscow) 20.07.90, No. 4096 - B90, 145pp.

5. N.K. Karapetyants, Kh.M. Murdaev and A.Ya. Yakubov, On isomorphism implemented by fractional integrals in generalized Hölder classes. (Russian) Dokl. Akad. Nauk SSSR 314(1990), 288-291. English translation: Sov. Math. Dokl. 42(1991), No.2, 446-450.

6. A.A. Kilbas, Potential type operators with power-logarithmic kernels in weighted Hölder spaces. (Russian) Izv. Akad. nauk Byel. SSR, Ser. Fiz. Mat. Nauk, (1978), No.2, 29-37.

7. E.R. Love, Fractional derivatives of imaginary order. J. London Math. Soc., Ser. 2(1971), 3, 241-259.

8. E.R. Love, Two index laws for fractional integrals and derivatives. $J$. Austral. Math. Soc. 14(1972), 385-410. 
9. A. Marchaud, Sur les derivees et sur les differences dans fonctions de variables reels. J. math. pures et appl. 6(1927), 337-425.

10. Kh.M. Murdaev, An estimate for continuity modulus of integrals and derivatives of fractional order. (Russian) Groznyi. Deposited in VINITI (Moscow) 14.06.85, No.4209.

11. Kh.M. Murdaev and S.G. Samko, Weighted estimates for continuity moduli of fractional integrals of functions having a prescribed continuity modulus with a weight. (Russian) Rostov-on-Don, Deposited in VINITI (Moscow) 11.05.86, No.3351-B, 42pp.

12. Kh.M. Murdaev and S.G. Samko, Mapping properties of fractional integro-differentiation in weighted generalized Hölder spaces $H_{0}^{\omega}(\rho)$ with the weight $\rho(x)=(x-a)^{\mu}(b-x)^{\nu}$. (Russian) Rostov-on-Don, Deposited in VINITI (Moscow) 11.05.86, No.3350-B, 25pp.

13. B.S. Rubin, Fractional integrals in Hölder spaces with a weight and potential type operators. (Russian) Izv. Akad. Nauk Arm. SSR, Ser. Mat. 9(1974), 308-324.

14. N.G. Samko, Weighted Zygmund estimate for the singular operator and a theorem on its boundedness in $H_{0}^{\omega}(\rho)$ in the case of general weights. (Russian) Rostov-on-Don, Deposited in VINITI (Moscow) 5.12.89, No.7559B89.

15. - - A singular operator and operators with a weak singularity in weighted generalized Hölder spaces. (Russian) Izv. Sev.-Kavk. Nauchn. Tsentra. Vysch. Scholy, Ser. Estestv. Nauk 1991, No.3, 46-69.

16. S.G. Samko, The space $I^{\alpha}\left(L_{p}\right)$ of fractional integrals and potential type operators. (Russian) Izv. Akad. nauk Armyan. SSR, Ser. Mat. 8(1973), 359-383.

17. S.G. Samko, A.A. Kilbas and O.I. Marichev, Fractional integrals and derivatives and some of their applications. (Russian) Minsk, Nauka $i$ Tekhnika, 1980, English translation: Gordon and Breach, to appear.

18. S.G. Samko and Kh.M. Murdaev, Weighted Zygmund estimates for fractional differentiation and integration and their applications. (Russian) Trudy Matem. Inst. Steklov. 180(1987), 197-198; English translation: Proc. Steklov Inst. Math. 1989, No.3, 233-235.

(Received 15.07.1993)

Author's address:

Rostov University

Department of Mathematics and Mechanics

105, ul. Bol'shaya Sadovaya,

Rostov-on-Don, 344711

Russia 